\title{
Neonatal MicroRNA Profile Determines Endothelial Function in Offspring of Hypertensive Pregnancies
}

\author{
Grace Z. Yu, Svetlana Reilly, Adam J. Lewandowski, Christina Y.L. Aye, Lisa J. Simpson, \\ Laura D. Newton, Esther F. Davis, Sha J. Zhu, Willow R. Fox, Anuj Goel, Hugh Watkins, \\ Keith M. Channon, Suzanne M. Watt, Theodosios Kyriakou, Paul Leeson
}

\begin{abstract}
Offspring of hypertensive pregnancies are at increased risk of developing hypertension in adulthood. In the neonatal period, they display endothelial cell dysfunction and altered microvascular development. MicroRNAs, as important endothelial cellular regulators, may play a role in this early endothelial dysfunction. Therefore, we identified differential microRNA patterns in endothelial cells from offspring of hypertensive pregnancies and determined their role in postnatal vascular cell function. Studies were performed on human umbilical vein endothelial cell samples from 57 pregnancies. Unbiased RNA sequencing identified 30 endothelial related microRNAs differentially expressed in human umbilical vein endothelial cells from hypertensive compared with normotensive pregnancies. Quantitative reverse transcription polymerase chain reaction confirmed a significant higher expression level of the top candidate, miR-146a. Combined miR-146a-targeted gene expression and pathway analysis revealed significant alterations in genes involved in inflammation, angiogenesis, and immune response in the same human umbilical vein endothelial cells. Elevated miR-146a expression level at birth identified cells with reduced ability for in vitro vascular tube formation, which was rescued by miR-146a inhibition. In contrast, miR-146a overexpression significantly reduced vascular tube formation in human umbilical vein endothelial cells from normotensive pregnancies. Finally, we confirmed that miR-146a levels at birth predicted in vivo microvascular development during the first 3 postnatal months. Offspring of hypertensive pregnancy have a distinct endothelial regulatory microRNA profile at birth, which is related to altered endothelial cell behavior and predicts patterns of microvascular development during the first 3 months of life. Modification of this microRNA profile in vitro can restore impaired vascular cell function. (Hypertension. 2018;72:937-945. DOI: 10.1161/HYPERTENSIONAHA.118.11343.) • Online Data Supplement
\end{abstract}

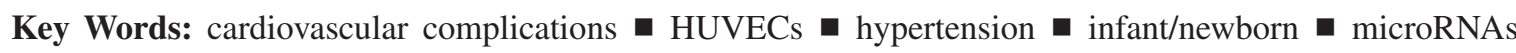
- microvasculature pregnancy

$\mathrm{O}$ ffspring of hypertensive pregnancies display changes in endothelial function from early in life $^{1}$ and are at an increased risk for hypertension in adulthood..$^{2-4}$ Circulating endothelial cells ${ }^{5}$ and umbilical vein-derived endothelial cells ${ }^{6}$ from neonates born to hypertensive pregnancies have altered cellular angiogenic capacity at birth. Furthermore, the poorer the function of the cells in vitro, the more likely the infant is to lose microvascular dermal density during the first postnatal months, as their circulation remodels. ${ }^{6}$ Microvascular dermal rarefaction has been linked to higher blood pressure in young adults born to pregnancy complications, ${ }^{7}$ and a primary abnormality in microvascular development presents a plausible mechanistic pathway to explain increased hypertensive risk for these offspring. ${ }^{8}$
MicroRNAs are a class of noncoding RNAs that are important regulators of cellular function. ${ }^{9}$ In recent years, several microRNAs have been shown to control aspects of endothelial cell behavior such as migration, proliferation, apoptosis, and vasculogenesis ${ }^{10}$ with certain microRNAs identified to be proangiogenic and others antiangiogenic. ${ }^{11-17}$ Previous studies have successfully demonstrated that alteration of endothelial microRNAs, using a knockdown approach, can have dramatic impacts on endothelial proliferation and tube formation in vitro. ${ }^{13,14}$ Stress conditions such as inflammation can also alter the regulation of microRNAs. ${ }^{16}$ It is, thus, plausible that in utero stress exposure related to hypertensive pregnancies may program alterations in offspring microRNA profile. Therefore, we hypothesized that

Received April 15, 2018; first decision May 1, 2018; revision accepted July 23, 2018.

From the Division of Cardiovascular Medicine, Radcliffe Department of Medicine (G.Z.Y., S.R., A.J.L., C.Y.L.A., L.J.S., L.D.N., E.F.D., W.R.F., A.G., H.W., K.M.C., T.K., P.L.), Stem Cell Research Laboratory, Nuffield Division of Clinical Laboratory Sciences, Radcliffe Department of Medicine (G.Z.Y., L.J.S., L.D.N., S.M.W.), Medical Sciences Division, Nuffield Department of Women's and Reproductive Health (C.Y.L.A.), and Wellcome Centre for Human Genetics (S.J.Z., A.G., T.K.), University of Oxford, United Kingdom; and NHS Blood and Transplant, Blood Donor Centre, John Radcliffe Hospital, Oxford, United Kingdom (G.Z.Y., L.J.S., L.D.N., S.M.W.).

The online-only Data Supplement is available with this article at https://www.ahajournals.org/doi/suppl/10.1161/HYPERTENSIONAHA. 118.11343.

Correspondence to Paul Leeson, Oxford Cardiovascular Clinical Research Facility, Division of Cardiovascular Medicine, British Heart Foundation (BHF) Centre of Research Excellence, Radcliffe Department of Medicine, University of Oxford, John Radcliffe Hospital, Oxford OX39DU, United Kingdom. Email paul.leeson@cardiov.ox.ac.uk

(C) 2018 The Authors. Hypertension is published on behalf of the American Heart Association, Inc., by Wolters Kluwer Health, Inc. This is an open access article under the terms of the Creative Commons Attribution Non-Commercial License, which permits use, distribution, and reproduction in any medium, provided that the original work is properly cited and is not used for commercial purposes.

Hypertension is available at https://www.ahajournals.org/journal/hyp 
endothelial cells from hypertensive pregnancy offspring would display a different pattern of microRNA expression compared with those derived from normotensive pregnancies. Furthermore, these patterns would predict vasculogenic capacity of endothelial cells and the in vivo development of the microvascular network in the neonate during the first 3 months of their postnatal life.

\section{Methods}

\section{Study Cohort and Sample Collection}

The data that support the findings of this study are available from the corresponding author on reasonable request. Mothers being cared for by Oxford University Hospitals NHS Foundation Trust between 2013 and 2015 were identified by their clinical care team and invited to take part in the Oxford Cardiovascular Tissue Bioresource program, coordinated by the Oxford Cardiovascular Clinical Research Facility and NHS Blood and Transplant. A clinical recruitment team approached mothers before delivery to seek consent for donation of tissue aiming for on average 2 to 3 participants every month. Mothers with either hypertensive or normotensive pregnancies were identified and approached in parallel to ensure balanced recruitment during a month. Rate of recruitment was controlled to ensure adequate time for sample preparation, including cord processing, cell isolation, and cell maintenance. Umbilical cords were collected immediately after delivery by a dedicated research cord collection team. All cords were processed within 12 hours of delivery. Human umbilical vein endothelial cells (HUVECs) were isolated and stored according to standard operating procedures (online-only Data Supplement). HUVECs were cultured in endothelial basal medium (EGM-2) supplemented with the EGM-2 bullet kit (catalog number: CC-3162; Lonza, UK). All cell cultures were maintained in humidified $5 \% \mathrm{CO}_{2}$ at $37^{\circ} \mathrm{C}$. All RNA expression analyses were performed using cells from passage 1 , whereas tube formation assays were performed with cells from passage 2 or 3 .

Pregnancy history including blood pressure levels were recorded for each participant from maternity records. Hypertensive pregnancies, including pregnancy-induced hypertension and preeclampsia, were defined according to the International Society for the Study of Hypertension in Pregnancy guidelines (definitions are available in the online-only Data Supplement). Normotensive pregnancy was also confirmed from case records, and if there was subsequent evidence of problems during pregnancy such as fetal growth restriction or glucose intolerance, samples were not included in analysis. The measurement of microvascular measures at birth and 3 months of age in the infants has been reported previously ${ }^{6}$ (detailed in online-only Data Supplement). All participants provided written consent with collection and subsequent experimental use of samples in accordance with approvals from appropriate National Research Ethics Committees (09/H0606/68, 07/H0606/148, 15/SC/0027, and 11/SC/0230).

\section{RNA Sequencing}

Total RNA $(1-5 \mu \mathrm{g})$ isolated from HUVECs $(\mathrm{n}=8)$ was processed using the NEBNext Small RNA Library Prep Set for Illumina (catalog number: E7330L) according to manufacturer's recommendations. The microRNA component of the library was enriched and quantified by real-time quantitative polymerase chain reaction (PCR). Sequencing was performed as $50 \mathrm{bp}$ single read on a GAIIx according to Illumina specifications using Cluster Generation Kit v4 and Sequencing Kit v4 (details are provided in the online-only Data Supplement). Quality control was performed to determine the proportion of reads mapping to genes and variation within the data set. The data were normalized using the R/BioConductor edgeR_2.99.0 package.

\section{Reverse Transcription and PCR}

TaqMan microRNA reverse transcription kit (Applied Biosystems; catalog number: 4366596) was used for cDNA synthesis according to the manufacturer's instructions. Expression of hsa-miR-146a in HUVECs was quantified using the microRNA TaqMan assay (Applied Biosystems; assay ID: 000468). Each PCR was performed in 3 technical replicates and expressed as fold change using the $2-\Delta \Delta \mathrm{Ct}$ method. ${ }^{18}$ Expression of miR-146a was normalized to the endogenous control RNU44 (Applied Biosystems; assay ID: 001094).

\section{Gene Expression and Network Analysis}

Total RNA of HUVECs was extracted using QIAGEN RNeasy Mini Kit (Qiagen, Germany) according to the manufacturer's instructions. RNA quality and abundance were assessed using a Nanodrop ND-1000 spectrophotometer (Nanodrop Technologies). Gene expression microarray analysis was conducted using Illunima HT-12v4 expression bead-chip arrays according to the manufacturer's protocol. The raw gene expression data were analyzed using the R/ BioConductor 'limma_3.26.9 package. Variance-stabilization and normalization algorithm was used for data normalization. Differential gene expression was determined using $t$ tests/ANOVA.

Using the experimentally validated microRNA-target interactions database (miRTarBase; http://mirtarbase.mbc.nctu.edu.tw), the expression level of the microRNA-targeted genes was extracted from the gene expression array results. We conducted a network enrichment analysis using Metacore based on combination of the selected microRNA-targeted genes and the expression results from the array experiment. Statistical significance was corrected using Bonferroni adjustment for the total number of genes in each network.

\section{Tube Formation and Cell Proliferation}

Angiogenic capacity was assessed by performing tube formation using growth factor-reduced Matrigel (BD Biosciences, UK). Cell proliferation was assessed using the CyQUANT NF Cell Proliferation Kit (Life Technologies) according to the manufacturer's instruction (detailed in online-only Data Supplement).

\section{Cell Transfection}

HUVECs were plated in a 6-well plate at $1 \times 10^{5}$ cells per well overnight. Cells were transfected with hsa-miR-146a-5p mimic $(10 \mathrm{nmol} / \mathrm{L})$, or hsa-miR-146a-5p hairpin inhibitor $(80 \mathrm{nmol} / \mathrm{L})$, or respective nontargeting controls (control \#1, Dharmacon) for 24 hours using Lipofectamine RNAiMAX (catalog number: 13778030; ThermoFisher) according to the manufacturer's instructions. Cells were then replaced with antibioticfree media for additional 48 hours and collected for functional assays. Transfections were visualized and optimized with fluorescently labeled mimic and inhibitor controls (Dharmacon). Transfection efficiency was $>90 \%$. The transfection of HUVECs with hsa-miR-146a inhibitor and mimic were confirmed by quantitative reverse transcription PCR, and immunoblotting was used to confirm alterations in a downstream protein level (details are provided in the online-only Data Supplement).

\section{In Vivo Microvascular Imaging}

Imaging of the axillary small vessel network was performed with Side Stream Dark Field imaging (Microscan; Microvision Medical, The Netherlands), as previously reported for neonates. ${ }^{19}$ Measurements were performed after birth and again at 3 months, on the same side, in a temperature-controlled room, with the infant at rest, either in their mother's arms or in a crib (details are provided in the online-only Data Supplement).

\section{Statistical Analysis}

Statistical analyses were conducted using SPSS version 22 and GraphPad Prism 6.0. Comparisons between continuous variables were performed using a 2-tailed, independent samples $t$ test for normally distributed variables and Mann-Whiney $U$ test for nonnormally distributed data. Levels of variables across the distribution of non-normally distributed variables were analyzed with the cohort ranked into thirds or tenths of the distribution. Comparison of categorical variables was performed using a $\chi^{2}$ test. Bivariable and multivariable regression models were performed using a forced entry method. Statistically significant variables from bivariable regression analysis were then included in a multivariable regression analysis. Pearson correlation was recorded for bivariable regression analysis. Standardized coefficients were used in the multiple regression analysis. Results are presented as mean \pm SD for normally distributed continuous variables, median (minimum-maximum) for non-normally 
distributed variables, and the number of observations (yes/no) with the percentage in each group for categorical variables. $P$ values $\leq 0.05$ were considered statistically significant.

\section{Results}

\section{Cohort Characteristics}

Samples from 57 pregnancies were used. Maternal and offspring demographic characteristics in the normotensive and hypertensive groups are presented in Table 1. Normotensive and hypertensive pregnancy groups were matched for maternal age, with no differences in smoking and alcohol consumption at time of pregnancy. Maternal body mass index (BMI), proportion of cesarean section, and blood pressure during pregnancy were significantly higher in the hypertensive mothers. Infants in the hypertensive group were on average 2 weeks more premature with a lower birth weight $z$ score. There were no differences in offspring sex distribution, head circumference, and Apgar score at 5 minutes between the 2 groups.

\section{MicroRNA Profiles in HUVECs From Normotensive and Hypertensive Pregnancy Offspring}

We selected 8 samples (4 hypertensive and 4 normotensive; Table S1 in the online-only Data Supplement), matched by

Table 1. Characteristics of Cohort

\begin{tabular}{|c|c|c|}
\hline Parameters & $\begin{array}{c}\text { Normotensive } \\
\quad(n=32)\end{array}$ & $\begin{array}{l}\text { Hypertensive } \\
\qquad(\mathrm{n}=25)\end{array}$ \\
\hline \multicolumn{3}{|l|}{ Mother } \\
\hline Maternal age, yr & $33 \pm 4$ & $33 \pm 5$ \\
\hline $\mathrm{BMI}$ at booking, $\mathrm{kg} / \mathrm{m}^{2}$ & $23.6 \pm 3.4$ & $28.1 \pm 6.5^{\star}$ \\
\hline Folic acid, n (\%) & $23(79)$ & $22(88)$ \\
\hline Smokers, n (\%) & $7(21)$ & $7(27)$ \\
\hline Alcohol this pregnancy, n (\%) & $3(9)$ & $3(12)$ \\
\hline Bleeding in early pregnancy, $\mathrm{n}(\%)$ & $3(9)$ & $4(15)$ \\
\hline Cesarean section, $\mathrm{n}(\%)$ & $5(15)$ & $17(71) \dagger$ \\
\hline Booking sBP, mm Hg & $110.5 \pm 10.7$ & $118.9 \pm 18.5 \ddagger$ \\
\hline Booking dBP, $\mathrm{mm} \mathrm{Hg}$ & $66.9 \pm 8.8$ & $71.7 .5 \pm 11.4$ \\
\hline Late gestation sBP, $\mathrm{mm} \mathrm{Hg}$ & $111.2 \pm 10.5$ & $124.5 \pm 16.6 \dagger$ \\
\hline Late gestation $\mathrm{dBP}, \mathrm{mm} \mathrm{Hg}$ & $65.1 \pm 7.7$ & $78.6 \pm 11.7 \dagger$ \\
\hline Highest sBP, mm Hg & $116.7 \pm 11.4$ & $143.5 \pm 19.7 \dagger$ \\
\hline Highest dBP, mm Hg & $70.8 \pm 8.3$ & $90.4 \pm 10.7 \dagger$ \\
\hline \multicolumn{3}{|l|}{ Offspring } \\
\hline Gestation age, wk & $39 \pm 1.7$ & $37 \pm 2.7 \dagger$ \\
\hline Birth weight, g & $3372.6 \pm 581.7$ & $2782.3 \pm 774.7^{*}$ \\
\hline Birth weight $z$ score & $0.36 \pm 0.8$ & $-0.44 \pm 1.0^{*}$ \\
\hline Males, n (\%) & $18(55)$ & $13(48)$ \\
\hline Head circumference, $\mathrm{cm}$ & $174.3 \pm 7.8$ & $175.7 \pm 9.4$ \\
\hline Apgar score (5 min) & $9.9 \pm 0.2$ & $9.7 \pm 1.0$ \\
\hline
\end{tabular}

Values are represented as mean \pm SD unless stated otherwise. BMI indicates body mass index; dBP, diastolic blood pressure; and SBP, systolic blood pressure.

${ }^{*} P<0.01$ by unpaired $t$ test.

$\dagger P<0.001$ by unpaired $t$ test.

$\ddagger P<0.05$ by unpaired $t$ test. maternal age and gestational age, based on a predefined RNA-sequencing experimental design for RNA-sequencing analysis with enrichment in microRNA profile to investigate the microRNA profile at birth. Thirty significantly altered microRNAs were identified between hypertensive and normotensive groups $(P<0.05$; Figure $1 \mathrm{~A})$. On the basis of a literature review, ${ }^{20}$ we identified 7 of these differentially expressed miRNAs (miR-146a, -27b, -31, -222 and -221, -130, and -92) in the hypertensive pregnancy cohort as having previously been reported to be regulators of endothelial responses (Table S2; Figure 1B). After false-discovery rate adjustment (Bonferroni correction based on the 20 preselected microRNA targets), miR-146a remained significantly upregulated with a 2.39-fold higher level in hypertensive HUVECs compared with the normotensive samples. We, therefore, took miR-146a forward as a candidate microRNA for validation and downstream functional investigation.

\section{Validation of MiR-146a in Hypertensive Pregnancy Offspring}

We confirmed (using quantitative reverse transcription PCR) in the full cohort of 57 participants that the level of miR146a in hypertensive HUVECs was 1.74-fold higher than in the normotensive group $(P=0.0006)$; miR-146a measured in a peripheral plasma sample was used as the reference sample for the comparison of hypertensive and normotensive samples (Figure 1C). We then studied other maternal and fetal risk factors that varied between hypertensive and normotensive pregnancy to determine whether there were any potential confounders in the association between hypertensive pregnancy and miR-146a fold change (Table S3). In bivariable regression analysis, gestational age trended toward an inverse correlation with miR-146a (coefficient $\mathrm{B}=-0.24 ; P=0.08)$. Therefore, we included gestational age, along with hypertensive pregnancy and maternal age, as independent variables in a multivariable regression model. This analysis demonstrated that hypertensive pregnancy was an independent predictor of miR-146a level, with gestational age showing no association with miR-146a (coefficient $\mathrm{B}=0.01 ; P=0.96$; Table 2).

\section{MiR-146a-Targeted Pathway Analysis in Endothelial Cells From Hypertensive Pregnancy Offspring}

To further determine the role of miR-146a to cellular function in endothelial cells from hypertensive pregnancies, we explored evidence of variation in the gene expression profile related to known targets for miR-146a using gene expression array data in the same cohort based on samples collected from 12 hypertensive and 12 normotensive mothers. After normalization, ANOVA comparative analysis identified 811 significantly differentially expressed genes $(P<0.05)$. Because the aim of this analysis was to focus on miR-146a-targeted genes, we limited the investigation to 97 targets, which were previously validated and extracted from the miRTarBase database (http:// mirtarbase.mbc.nctu.edu.tw), with details in the Methods section. Among the 97 selected miR-146a gene targets, 67 targets were detected in the expression array results (Table S4). Gene enrichment network analysis using Metacore was performed using the combined miR-146a-targeted gene list and gene 


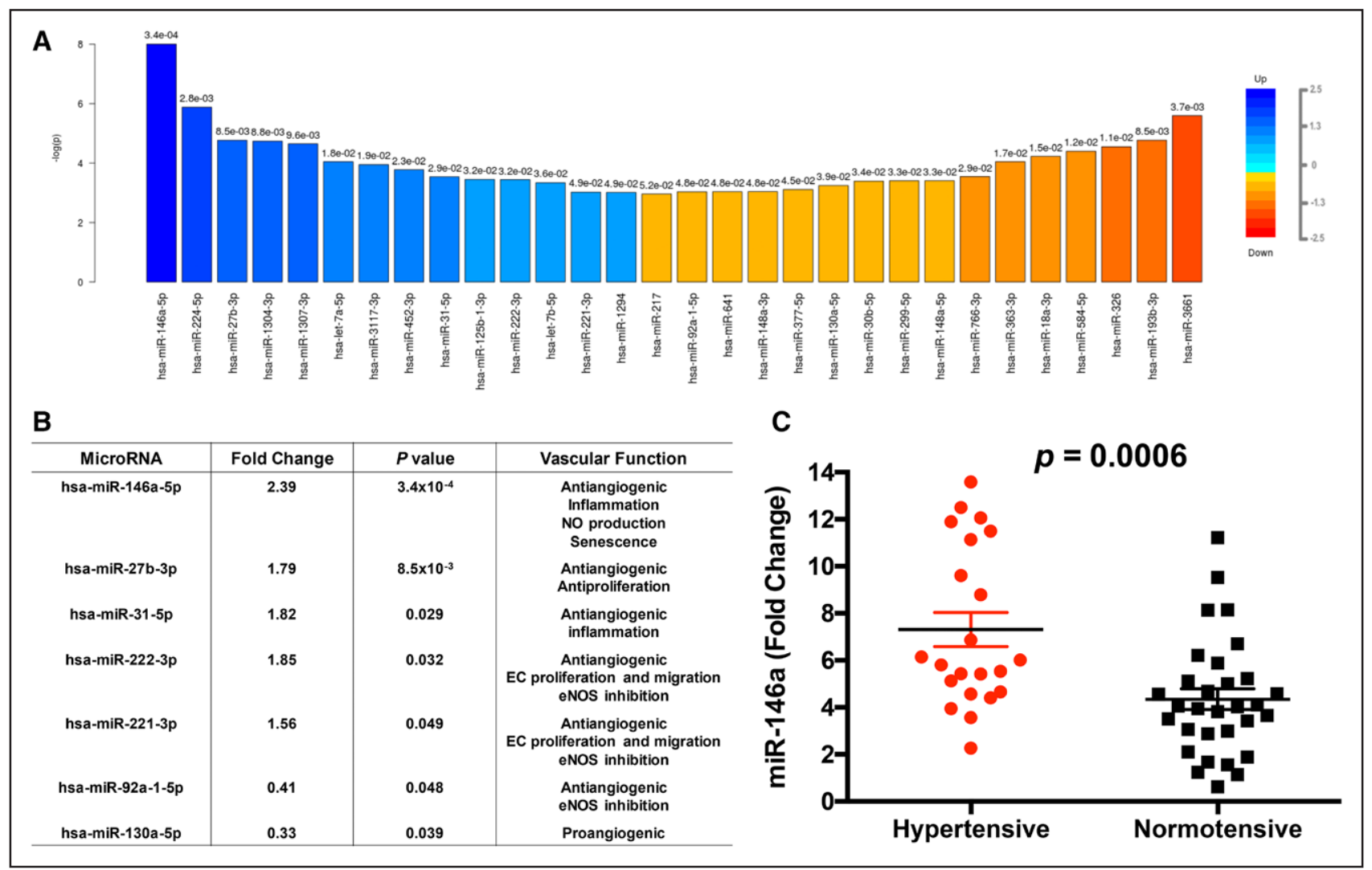

Figure 1. MicroRNA profiles in human umbilical vein endothelial cells (HUVECs) from normotensive and hypertensive pregnancy offspring. A, Top 30 differently expressed microRNAs in HUVECs derived from hypertensive and normal pregnancies ( $\mathrm{n}=8$ ). Fold change is indicated by color gradient, with darker colors representing higher fold change, where blue represents upregulation of microRNA targets and orange represents downregulation of microRNA targets in the comparison. Numbers above the fold change color bars represent the $P$ value obtained from the $t$ test comparison between groups. B, Validation in RNA sequencing of previously reported microRNAs in endothelial cells. EC indicates endothelial cell; and eNOS, endothelial nitric oxide synthase. C, MiR146 a expression (quantitative reverse transcription polymerase chain reaction) in HUVECs isolated from hypertensive pregnancy ( $\mathrm{n}=25$ ) and normotensive pregnancy $(n=32)$. Both groups were compared with a baseline, that is, miR-146a level measured in a peripheral blood sample of a healthy individual; $P$ value is obtained by unpaired $t$ test.

expression profile. The combined approach has been shown to be instrumental in detecting group effects in microRNA expression and related target gene sets. ${ }^{21} \mathrm{~A}$ summary of the miR-146a-targeted selection process is shown in Figure 2A. Using this selection process, 44 networks were identified with statistically significant associations $(P<0.05)$ after correcting for the total number of genes in each pathway (Figure S1). Among all miR-146a-targeted networks, the top 10 networks relate to inflammation, angiogenesis, and immune response (Figure 2B). Network objects are listed in Figure S2 showing the total number of networks that each object is involved in and ranking of each object among all network maps.

Table 2. Multivariable Regression Coefficients for Maternal and Fetal Risk Factors and MiR-146a

\begin{tabular}{|l|c|c|}
\hline Parameters & $\begin{array}{c}\text { Coefficient B } \\
\text { (miR-146a Fold Change) }\end{array}$ & PValue \\
\hline $\begin{array}{l}\text { Maternal hypertension } \\
\text { during pregnancy }\end{array}$ & 3.25 & $\mathbf{0 . 0 0 2}$ \\
\hline Maternal age, yr & -0.13 & 0.14 \\
\hline Gestational age, wk & 0.01 & 0.96 \\
\hline
\end{tabular}

B-unstandardized coefficient with 95\% confidence interval. Bold formatting represents $P<0.05$.

\section{MiR-146a Regulates Endothelial Tube Formation in Offspring of Hypertensive Pregnancy}

To establish whether manipulation of miR-146a level could directly influence the vascular phenotype of cells from offspring of hypertensive pregnancy, we transfected HUVECs derived from hypertensive and normotensive pregnancies with miR-146a mimic and hairpin inhibitor for 24 hours, with the average transfection efficiency greater than $90 \%$. Subsequent quantitative reverse transcription PCR in HUVECs confirmed a significant increase in miR-146a level after transfection with miR-146a mimic (the effect of miR-146a mimic on a downstream target protein was confirmed by immunoblotting) and a $68 \%$ reduction in miR-146a after transfection with miR-146a hairpin inhibitor (Figure S3).

Functionally, overexpression of miR-146a reduced the total tubule length $(17627 \pm 958$ versus $13995 \pm 1295 ; P=0.05)$ and number of branching points $(133 \pm 5$ versus $100 \pm 11$; $P=0.03$ ) in normal HUVECs, although similar responses were not apparent in already dysfunctional HUVECs derived from hypertensive pregnancies (Figure 3A). By contrast, inhibition of miR-146a restored the total tubule length $(13339 \pm 789$ versus $16064 \pm 644 ; P=0.04)$ and branching points $(93 \pm 8$ versus $128 \pm 3 ; P=0.008)$ in the hypertensive group only, indicating that inhibition of miR-146a in HUVECs derived from hypertensive pregnancy, to some extent, is capable of recovering 


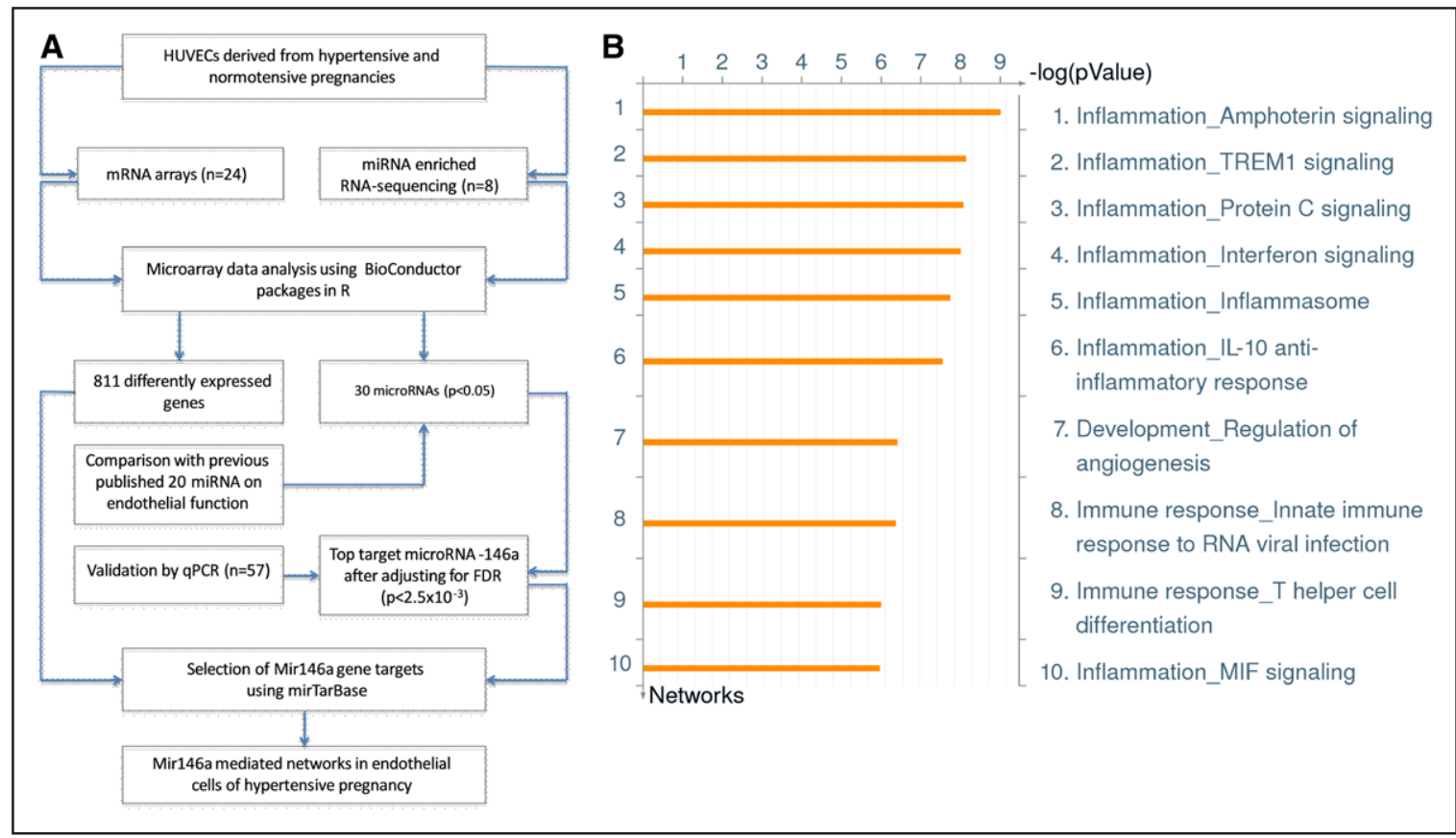

Figure 2. MiR-146a-targeted pathway analysis in endothelial cells. A, Schematic workflow to identify miR-146a-targeted pathways using a combined mRNA and microRNA approach in hypertensive pregnancy. B, Ranking of miR-146a-targeted enrichment networks in vascular development. Network enrichment analysis was performed using Metacore. HUVECs indicates human umbilical vein endothelial cells; and qPCR, quantitative polymerase chain reaction.

angiogenic capacity compared with cells from the normotensive group. Indeed, tube formation was no longer significantly different between these 2 groups ( $P=0.26$; Figure $3 \mathrm{~B})$. Similar to the tube formation, overexpression of miR-146a in HUVECs from hypertensive pregnancies resulted in reduced cell proliferation that was ameliorated by miR-146a inhibition (66593 \pm 4680 versus $80970 \pm 5247 ; P=0.06$; Figure 3).

\section{Association of MiR-146a Expression With In Vitro and In Vivo Vessel Formation}

To determine whether levels of miR-146a were relevant to the in vivo and in vitro microvascular responses observed in offspring born to hypertensive pregnancy, we correlated levels with endothelial capacity for tube formation and proliferation in 49 participants. The level of miR-146a expression was negatively associated with total tubule length $(P=0.001)$, branch points $(P=0.01)$, and cell proliferation $(P=0.0$; Figure 4A; Figure $S 4)$. Further, there was a graded association between miR-146a levels and dermal microvascular density in the same individuals (10 hypertensive and 18 normotensive pregnancies) at 3 months postnatal age (Figure 4B), with higher miR-146a levels related to reduced microvascular density at 3 months $(P=0.003$ for total vessel density; $P=0.005$ for De Backer score).

\section{Discussion}

Our study shows that endothelial cells from neonates born after a hypertensive pregnancy have significantly altered expression levels of microRNAs compared with equivalent cells from offspring of normotensive pregnancies. Specific microRNAs known to be relevant to endothelial cell phenotype/function, and, in particular, miR-146a, differ significantly in endothelium of hypertensive pregnancy offspring, with associated changes in the expression level of their gene targets. These differences in the microRNA profile are also associated with an impaired in vitro endothelial cell tubulogenesis phenotype in offspring of hypertensive pregnancies, which can be induced by miR-146a overexpression and rescued by miR-146a inhibition. Finally, we demonstrate that microRNA expression profile at birth in the HUVECs of an offspring can predict the pattern of microvascular development in that neonate during the next 3 months of life. Although the molecular mechanism through which miR-146a modulates vasculogenesis has not been explored, these results support a regulatory role of microRNAs in endothelial dysfunction seen in offspring of hypertensive pregnancy.

The early postnatal period is considered a critical developmental window for the microvasculature. Our previous work demonstrated that endothelial cellular behavior at birth predicts early postnatal microvascular remodeling. ${ }^{6}$ Posttranscriptional regulation of gene expression by microRNAs is known to be important to several endothelial phenotypes, ${ }^{20}$ and, therefore, we were able to increase the efficiency of our study by using prior knowledge from the literature to focus on candidate endothelial microRNAs. A drawback of this approach is that it did not allow us to probe for potentially relevant novel microRNA targets. Nevertheless, the 7 validated microRNAs have previously been linked with changes in angiogenesis and endothelial cell tubulogenesis and were confirmed in our study. ${ }^{11,13-17,22}$ Our findings are, therefore, consistent with previous evidence for these microRNAs and, for the first time, implicates them as mediators of the antiangiogenic state previously reported in endothelial colony-forming cells ${ }^{5,23}$ and umbilical-derived endothelial cells ${ }^{6}$ collected in neonates born after a hypertensive pregnancy.

The greatest difference in microRNA expression levels was for miR-146a, a microRNA that has previously 

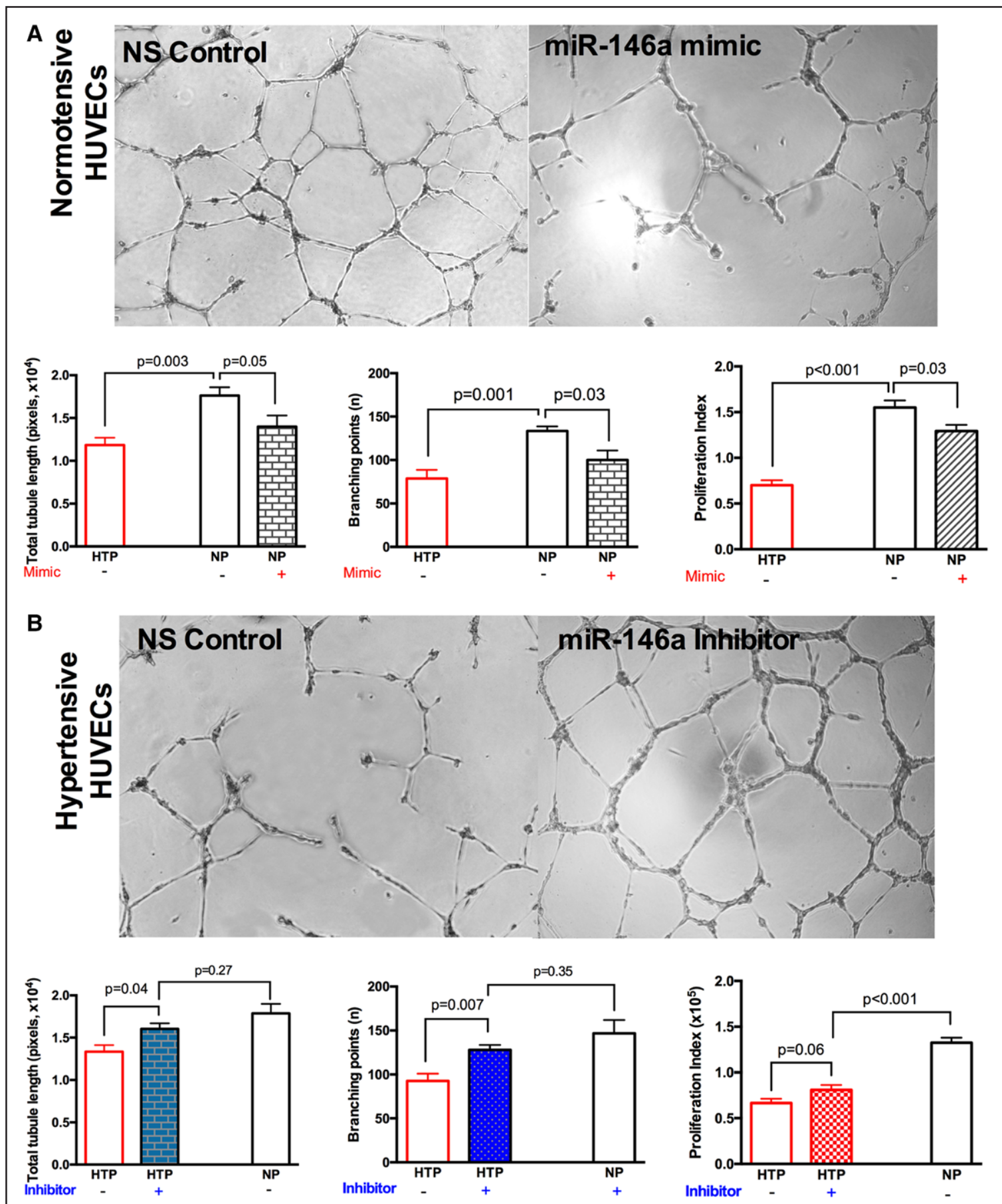

Figure 3. MiR-146a regulates endothelial tube formation in human umbilical vein endothelial cells (HUVECs). A and B, Representative images of HUVECs in growth factor-reduced Matrigel after transfection with miR-146a mimic, miR-146a inhibitor, or nontargeting (NS) control. Quantification of tubule formation (total tubule length and branching points) and proliferation index of HUVECs are summarized in respective bar graphs ( $n=10)$. HTP indicates hypertensive pregnancy; and NP, normotensive pregnancy. $P$ value is obtained by unpaired $t$ test.

been studied in relation to angiogenesis in cancer, ${ }^{24,25}$ innate immune signaling, ${ }^{12,26}$ and inflammatory pathways. ${ }^{22,27}$ MiR$146 \mathrm{a}$ is upregulated, as part of a feedback loop, on activation of a proinflammatory transcriptional program related to $\mathrm{NF}-\mathrm{kB}$ (nuclear factor $\kappa$-light-chain-enhancer of activated $\mathrm{B}$ cells) signaling. ${ }^{26,28}$ After removal of proinflammatory cytokines, elevated levels of miR-146a are sustained for several days, suggesting the maintenance of a prolonged inflammatory memory. ${ }^{22}$ Using gene expression profiling in endothelial cells from normal and hypertensive offspring, we confirmed variation in miR-146a target pathways related to inflammation. Severe hypertensive pregnancies, such as preeclampsia, commonly induce a proinflammatory state in the mother. ${ }^{29}$ It is, therefore, plausible that the elevated levels of miR-146a levels in the neonate reflect a persistent upregulation after exposure of the fetus to an inflammatory insult during pregnancy. However, other studies suggest that the fetus is relatively protected from the adverse maternal circulating milieu, ${ }^{30}$ except in 


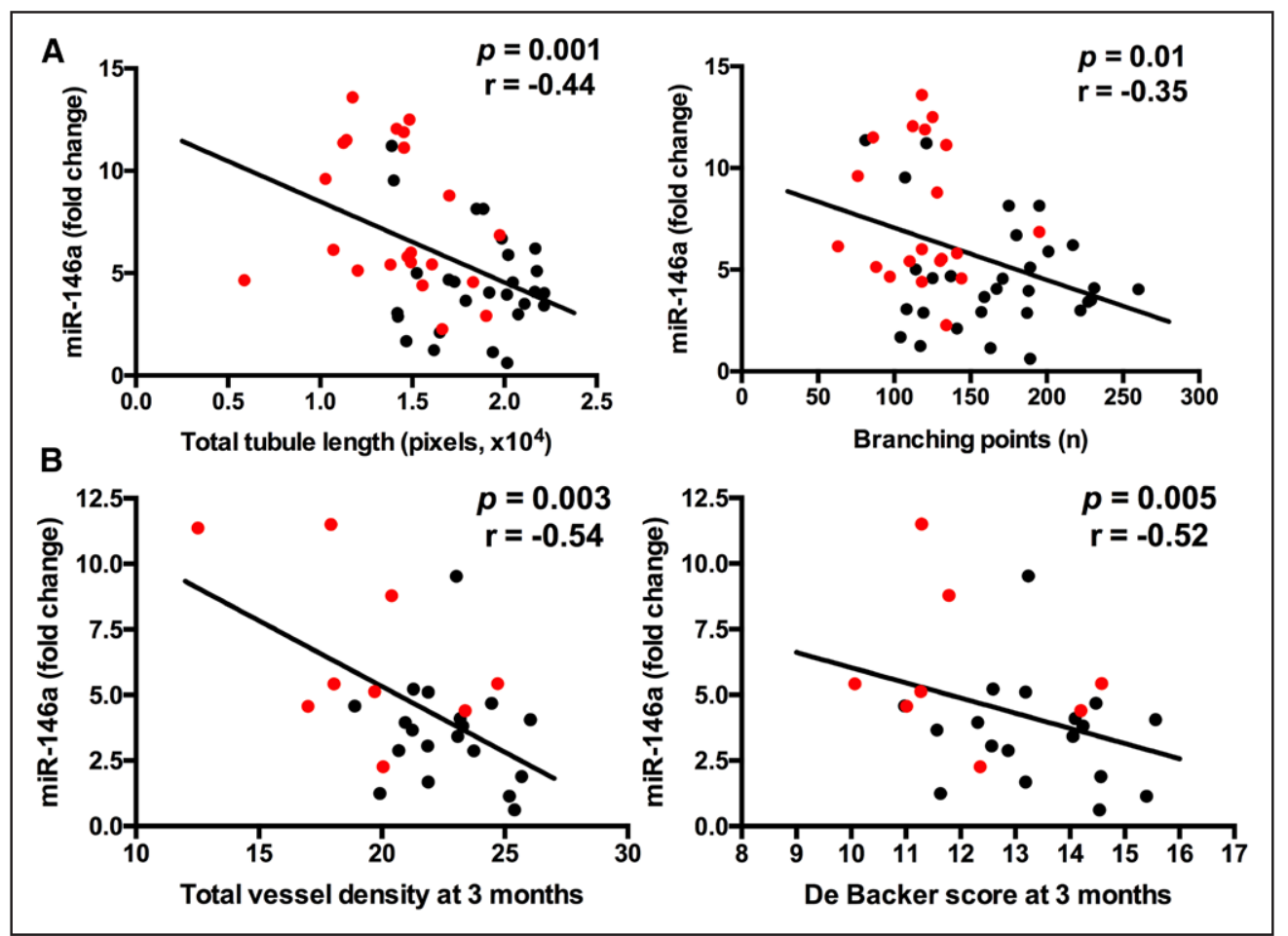

Figure 4. Association of miR-146a expression with in vitro and in vivo vessel formation. A, Correlation of miR-146a level with in vitro tubule network measurements (total tubule length and branching points) using paired samples from the same infants $(n=49)$. Human umbilical vein endothelial cells (HUVECs) were extracted from umbilical cords within $12 \mathrm{~h}$ after delivery. MicroRNAs were measured in HUVECs at passage 1, whereas tubule measurements were measured in HUVECs at passages 2 and 3. B, Correlation of miR-146a level with in vivo microvasculature at 3 postnatal months using paired samples from the same infant $(n=28)$. MiR-146a levels were obtained from quantitative reverse transcription PCR.

the most severe pregnancy complications with associated fetal growth restriction. This was not a predominant finding in our participant population, and we found miR-146a to have antiangiogenic properties, in contrast to the proangiogenic miR146a response seen during inflammation. ${ }^{25}$ HUVECs exposed to a major inflammatory insult such as lipopolysaccharide ${ }^{29}$ or when grown in an adverse metabolic state, such as seen in diabetes mellitus, ${ }^{28}$ can be induced to increase miR-146a expression and enhance their tubule formation.

Our cells were maintained in a neutral environment, and differences in miR-146a were already evident at baseline. Endothelial colony-forming cells from patients with coronary artery disease similarly have increased levels of miR-146a and exhibit reduced angiogenic capacity. ${ }^{31}$ Consistent with our observations, inhibition of miR-146a restores endothelial tubulogenesis in endothelial colony-forming cells from patients with coronary artery disease, via a distinct RhoJdependent mechanism. ${ }^{31}$ miR-146a also inhibits neoformation of blood vessels in a model of choroidal lesions, and blocking miR-146a leads to longer tube formation in an ex vivo aortic ring angiogenic assay. ${ }^{14}$ An equivalent, antiangiogenic impact of miR-146a has been associated with other pregnancy complications. ${ }^{14}$ Halkein et $\mathrm{al}^{14}$ showed that exosomal miR146a levels are increased in peripartum cardiomyopathy, and myocardial uptake results in apoptosis and reduced angiogenesis. Furthermore, overexpression of miR-146a was found to repress proliferation and apoptosis in vitro.

We grouped results from offspring born to both pregnancy-induced hypertension and preeclampsia as we have previously shown these pregnancy complications are both associated with differences in endothelial cell behavior in the neonates. ${ }^{6}$ However, the sample size was not sufficient to determine whether there are graded effects between offspring born to these 2 distinct hypertensive pregnancy complications. Maternal miR-146a levels are also altered during pregnancy, in a dose-dependent manner, in response to tobacco smoke exposure. ${ }^{32,33}$ Therefore, we undertook a regression analysis to understand whether other pregnancy factors such as maternal BMI or fetal factors such as gestational age or growth restriction may be confounders for the association we found between hypertensive pregnancy and offspring endothelial microRNA levels. This indicated that the main predictor of miR-146a levels in the neonate was a history of hypertensive pregnancy rather than other factors such as preterm birth. Small group differences in maternal BMI were evident, both in the smaller RNA-sequencing cohort and full cohort. However, we found no relationship between maternal BMI and miR-146a levels in our cohort, suggesting the relationship between hypertensive pregnancies and miR-146a in the offspring is independent of maternal BMI. Nevertheless, we did not have information on some factors such as maternal metabolic function, and given the differences in maternal BMI between study groups, future studies will be needed to assess how metabolic alterations and lifestyle alter miR-146a level. It has recently been suggested that offspring born to normotensive pregnancies, but whose mother has a history of hypertensive pregnancy, may also be predisposed to hypertension $^{34}$; thus, it will be interesting to see whether altered microRNA profiles are seen in circulating endothelial cells at other points in life or in siblings of affected families. Future 
studies will then be able to assess the relative contribution of family history of hypertension to these alterations in endothelial phenotype in the offspring.

Expression array analysis was performed in cells at passage 1 , whereas in vitro tube formation assays were performed with cells from passage 2 or 3 . It has previously been shown that there is a slight shift in microRNA expression from passage 1 to 2 or $3,{ }^{35}$ and, therefore, this may have weakened any association between expression levels and the in vitro assay. Furthermore, microRNA-146a levels were validated using the $\Delta \Delta \mathrm{Ct}$ method for quantification, which makes several assumptions that are addressed using other approaches such as Cq quantification. ${ }^{36}$ Nevertheless, multiple quality control steps were taken to ensure high-quality PCR, and we showed rescue of vasculogenic potential by inhibition of miR-146a. It will be of interest to explore in experimental models whether miR-146a can also alter postnatal microvascular development in vivo. Our data in humans are necessarily associative, and we cannot prove causality for associations between endothelial capacity and microvascular remodeling. However, the graded nature of the association suggests that the endothelial cell phenotype is a biomarker at birth that usefully predicts patterns of vascular development. It is possible that manipulation of microRNA profile during a critical postnatal window of development could have longterm benefits for vascular development and, hence, alter risk for later development of hypertension. Because microRNAs tend to have multiple gene targets and are interdependent, it is possible that the effects on tubulogenesis involve multiple pathways that can be targeted distinctly, and experimental models will be of value to explore molecular signals.

\section{Perspectives}

This study has shown for the first time that endothelial cells from neonates born after a hypertensive pregnancy have a distinct microRNA profile associated with an altered endothelial phenotype. The most upregulated microRNA was miR-146a, and its levels predict both in vivo and in vitro vascular phenotypes seen in hypertensive pregnancy offspring. Furthermore, inhibition of miR-146a resulted in rescue of the antiangiogenic state. These results suggest that microRNAs are central to the abnormal vascular state observed in offspring of hypertensive pregnancy.

\section{Acknowledgments}

We are grateful to all the pregnant woman and babies who participated in this study. We thank the High-Throughput Genomics Group at the Wellcome Trust Centre for Human Genetics (funded by Wellcome Trust grant reference $090532 / \mathrm{Z} / 09 / \mathrm{Z}$ ) for the generation of the gene expression and RNA-sequencing data.

\section{Sources of Funding}

This work was supported by the British Heart Foundation (BHF; grants FS/06/024 and FS/11/65/28865 to P. Leeson; RG/13/1/30181 and $\mathrm{CH} / 16 / 1 / 32013$ to K.M. Channon). Support was also provided by the National Institute for Health Research Oxford Biomedical Research Centre and by grants to S. Watt by National Health Service Blood and Transplant and the National Institute of Health Research under its Programme Grants Scheme (RP-PG-0310-1003 and RP-PG-0310-1001).

\section{Disclosures}

None.

\section{References}

1. Lazdam M, de la Horra A, Pitcher A, Mannie Z, Diesch J, Trevitt C, Kylintireas I, Contractor H, Singhal A, Lucas A, Neubauer S, Kharbanda R, Alp N, Kelly B, Leeson P. Elevated blood pressure in offspring born premature to hypertensive pregnancy: is endothelial dysfunction the underlying vascular mechanism? Hypertension. 2010;56:159-165. doi: 10.1161/HYPERTENSIONAHA.110.150235

2. Bellamy L, Casas JP, Hingorani AD, Williams DJ. Pre-eclampsia and risk of cardiovascular disease and cancer in later life: systematic review and meta-analysis. BMJ. 2007;335:974. doi: 10.1136/bmj.39335.385301.BE

3. Davis EF, Lazdam M, Lewandowski AJ, Worton SA, Kelly B, Kenworthy Y, Adwani S, Wilkinson AR, McCormick K, Sargent I, Redman C, Leeson P. Cardiovascular risk factors in children and young adults born to preeclamptic pregnancies: a systematic review. Pediatrics. 2012;129:e1552e1561. doi: 10.1542/peds.2011-3093

4. Davis EF, Lewandowski AJ, Aye C, Williamson W, Boardman H, Huang RC, Mori TA, Newnham J, Beilin LJ, Leeson P. Clinical cardiovascular risk during young adulthood in offspring of hypertensive pregnancies: insights from a 20-year prospective follow-up birth cohort. BMJ Open. 2015;5:e008136. doi: 10.1136/bmjopen-2015-008136

5. Ligi I, Simoncini S, Tellier E, et al. A switch toward angiostatic gene expression impairs the angiogenic properties of endothelial progenitor cells in low birth weight preterm infants. Blood. 2011;118:1699-1709. doi: 10.1182/blood-2010-12-325142

6. Yu GZ, Aye CY, Lewandowski AJ, et al. Association of maternal antiangiogenic profile at birth with early postnatal loss of microvascular density in offspring of hypertensive pregnancies. Hypertension. 2016;68:749759. doi: 10.1161/HYPERTENSIONAHA.116.07586

7. Lewandowski AJ, Davis EF, Yu G, Digby JE, Boardman H, Whitworth P, Singhal A, Lucas A, McCormick K, Shore AC, Leeson P. Elevated blood pressure in preterm-born offspring associates with a distinct antiangiogenic state and microvascular abnormalities in adult life. Hypertension. 2015;65:607-614. doi: 10.1161/HYPERTENSIONAHA.114.04662

8. Davis EF, Newton L, Lewandowski AJ, Lazdam M, Kelly BA, Kyriakou T, Leeson P. Pre-eclampsia and offspring cardiovascular health: mechanistic insights from experimental studies. Clin Sci (Lond). 2012;123:5372. doi: 10.1042/CS20110627

9. Morales Prieto DM, Markert UR. MicroRNAs in pregnancy. J Reprod Immunol. 2011;88:106-111. doi: 10.1016/j.jri.2011.01.004

10. Wu F, Yang Z, Li G. Role of specific microRNAs for endothelial function and angiogenesis. Biochem Biophys Res Commun. 2009;386:549-553. doi: 10.1016/j.bbrc.2009.06.075

11. Bonauer A, Carmona G, Iwasaki M, et al. MicroRNA-92a controls angiogenesis and functional recovery of ischemic tissues in mice. Science. 2009;324:1710-1713. doi: 10.1126/science.1174381

12. Chan EK, Ceribelli A, Satoh M. Microrna-146a in autoimmunity and innate immune responses. Ann Rheum Dis. 2013;72(suppl 2):ii90-ii95. doi: 10.1136/annrheumdis-2012-202203

13. Chen Y, Gorski DH. Regulation of angiogenesis through a microRNA (miR-130a) that down-regulates antiangiogenic homeobox genes GAX and HOXA5. Blood. 2008;111:1217-1226. doi: 10.1182/blood-2007-07-104133

14. Halkein J, Tabruyn SP, Ricke-Hoch M, Haghikia A, Nguyen NQ, Scherr M, Castermans K, Malvaux L, Lambert V, Thiry M, Sliwa K, Noel A, Martial JA, Hilfiker-Kleiner D, Struman I. MicroRNA-146a is a therapeutic target and biomarker for peripartum cardiomyopathy. J Clin Invest. 2013;123:2143-2154. doi: 10.1172/JCI64365

15. Poliseno L, Tuccoli A, Mariani L, Evangelista M, Citti L, Woods K, Mercatanti A, Hammond S, Rainaldi G. MicroRNAs modulate the angiogenic properties of HUVECs. Blood. 2006;108:3068-3071. doi: 10.1182/blood-2006-01-012369

16. Suárez Y, Wang C, Manes TD, Pober JS. Cutting edge: TNF-induced microRNAs regulate TNF-induced expression of E-selectin and intercellular adhesion molecule-1 on human endothelial cells: feedback control of inflammation. J Immunol. 2010;184:21-25. doi: 10.4049/jimmunol. 0902369

17. Ye J, Wu X, Wu D, Wu P, Ni C, Zhang Z, Chen Z, Qiu F, Xu J, Huang J. miRNA-27b targets vascular endothelial growth factor $\mathrm{C}$ to inhibit tumor progression and angiogenesis in colorectal cancer. PLoS One. 2013;8:e60687. doi: 10.1371/journal.pone.0060687

18. Livak KJ, Schmittgen TD. Analysis of relative gene expression data using real-time quantitative PCR and the 2(-Delta Delta C(T)) method. Methods. 2001;25:402-408. doi: 10.1006/meth.2001.1262

19. Genzel-Boroviczény O, Strötgen J, Harris AG, Messmer K, Christ F. Orthogonal polarization spectral imaging (OPS): a novel method to 
measure the microcirculation in term and preterm infants transcutaneously. Pediatr Res. 2002;51:386-391. doi: 10.1203/00006450-200203000-00019

20. Yamakuchi M. MicroRNAs in vascular biology. Int $J$ Vasc Med. 2012;2012:794898. doi: 10.1155/2012/794898

21. Artmann S, Jung K, Bleckmann A, Beissbarth T. Detection of simultaneous group effects in microRNA expression and related target gene sets. PLoS One. 2012;7:e38365. doi: 10.1371/journal.pone.0038365

22. Cheng HS, Sivachandran N, Lau A, Boudreau E, Zhao JL, Baltimore D, Delgado-Olguin P, Cybulsky MI, Fish JE. MicroRNA-146 represses endothelial activation by inhibiting pro-inflammatory pathways. EMBO Mol Med. 2013;5:1017-1034. doi: 10.1002/emmm.201202318

23. Muñoz-Hernandez R, Miranda ML, Stiefel P, Lin RZ, Praena-Fernández JM, Dominguez-Simeon MJ, Villar J, Moreno-Luna R, Melero-Martin JM. Decreased level of cord blood circulating endothelial colonyforming cells in preeclampsia. Hypertension. 2014;64:165-171. doi: 10.1161/HYPERTENSIONAHA.113.03058

24. Huang WT, Cen WL, He RQ, Xie Y, Zhang Y, Li P, Gan TQ, Chen G, Hu XH. Effect of miR-146a-5p on tumor growth in NSCLC using chick chorioallantoic membrane assay and bioinformatics investigation. Mol Med Rep. 2017;16:8781-8792. doi: 10.3892/mmr.2017.7713

25. Zhu K, Pan Q, Zhang X, et al. MiR-146a enhances angiogenic activity of endothelial cells in hepatocellular carcinoma by promoting PDGFRA expression. Carcinogenesis. 2013;34:2071-2079. doi: 10.1093/carcin/bgt160

26. Taganov KD, Boldin MP, Chang KJ, Baltimore D. NF-kappaB-dependent induction of microRNA miR-146, an inhibitor targeted to signaling proteins of innate immune responses. Proc Natl Acad Sci USA. 2006;103:12481-12486. doi: 10.1073/pnas.0605298103

27. Yang K, He YS, Wang XQ, Lu L, Chen QJ, Liu J, Sun Z, Shen WF. MiR146a inhibits oxidized low-density lipoprotein-induced lipid accumulation and inflammatory response via targeting toll-like receptor 4. FEBS Lett. 2011;585:854-860. doi: 10.1016/j.febslet.2011.02.009

28. Bhaumik D, Scott GK, Schokrpur S, Patil CK, Campisi J, Benz CC. Expression of microRNA-146 suppresses NF-kappaB activity with reduction of metastatic potential in breast cancer cells. Oncogene. 2008;27:5643-5647. doi: 10.1038/onc.2008.171

29. Lazdam M, Davis EF, Lewandowski AJ, Worton SA, Kenworthy Y, Kelly B, Leeson P. Prevention of vascular dysfunction after preeclampsia: a potential long-term outcome measure and an emerging goal for treatment. J Pregnancy. 2012;2012:704146. doi: 10.1155/2012/704146

30. Boutsikou T, Mastorakos G, Kyriakakou M, Margeli A, Hassiakos D, Papassotiriou I, Kanaka-Gantenbein C, Malamitsi-Puchner A. Circulating levels of inflammatory markers in intrauterine growth restriction. Mediators Inflamm. 2010;2010:790605. doi: 10.1155/2010/790605

31. Chang TY, Tsai WC, Huang TS, Su SH, Chang CY, Ma HY, Wu CH, Yang CY, Lin CH, Huang PH, Cheng CC, Cheng SM, Wang HW. Dysregulation of endothelial colony-forming cell function by a negative feedback loop of circulating miR-146a and -146b in cardiovascular disease patients. PLoS One. 2017;12:e0181562. doi: 10.1371/journal.pone.0181562

32. Maccani MA, Avissar-Whiting M, Banister CE, McGonnigal B, Padbury $\mathrm{JF}$, Marsit CJ. Maternal cigarette smoking during pregnancy is associated with downregulation of miR-16, miR-21, and miR-146a in the placenta. Epigenetics. 2010;5:583-589.

33. Tsochandaridis M, Nasca L, Toga C, Levy-Mozziconacci A. Circulating microRNAs as clinical biomarkers in the predictions of pregnancy complications. Biomed Res Int. 2015;2015:294954. doi: 10.1155/2015/294954

34. Yu GZ, Leeson P. Hypertension: hypertension in pregnancy: a risk factor for the whole family? Nat Rev Nephrol. 2017;13:326-327. doi: 10.1038/nrneph.2017.54

35. Kuosmanen SM, Kansanen E, Sihvola V, Levonen AL. MicroRNA profiling reveals distinct profiles for tissue-derived and cultured endothelial cells. Sci Rep. 2017;7:10943. doi: 10.1038/s41598-017-11487-4

36. Bustin SA, Benes V, Garson JA, Hellemans J, Huggett J, Kubista M, Mueller R, Nolan T, Pfaffl MW, Shipley GL, Vandesompele J, Wittwer CT. The MIQE guidelines: minimum information for publication of quantitative real-time PCR experiments. Clin Chem. 2009;55:611-622. doi: 10.1373/clinchem.2008.112797

\section{Novelty and Significance}

\section{What Is New?}

- Neonates born after hypertensive pregnancies have a distinct endothelial regulatory microRNA profile compared with offspring of normotensive pregnancies.

- The most altered microRNA, microRNA-146a, predicts microvascular development in early postnatal life.

\section{What Is Relevant?}

- Offspring of hypertensive pregnancies have reduced microvascular density from early postnatal life and are at increased risk of hypertension by young adulthood.
- Abnormal microvascular development increases risk for hypertension and may, therefore, be an important target for cardiovascular risk reduction.

\section{Summary}

The microRNA expression pattern in umbilical endothelial cells in offspring of hypertensive pregnancies is altered and relates with a more rapid loss of in vivo microvasculature in early postnatal life. Modification of microRNAs could improve microvascular formation and reduced hypertension risk. 